\title{
Patrón de la regeneración tras cortas a hecho en dos tiempos sobre masas de repoblación de Pinus pinaster Ait. con presencia variable de frondosas (Sierra Madrona)
}

Regeneration pattern after seed-tree method fellings in Pinus pinaster Ait. reforestations with variable presence of broad-leaved species

(Sierra Madrona)

de Frutos, S. ${ }^{*}$; del Río, M. ${ }^{2}$; Ruiz-Peinado, R. ${ }^{2}$; Bravo-Fernández, J.A. ${ }^{1}$

${ }^{1}$ Departamento de Sistemas y Recursos Naturales. Escuela Técnica Superior de Ingeniería de Montes,

Forestal y del Medio Natural. Universidad Politécnica de Madrid.

C/ José Antonio Novais 10. 28040 Madrid

${ }^{2}$ Centro de Investigación Forestal (CIFOR-INIA). Ctra. de La Coruña, km. 7,5. 28040 Madrid 


\section{Resumen}

El contexto actual de cambio global obliga a realizar una selvicultura adaptativa en todo tipo de masas forestales, pero la particular situación de miles de hectáreas de repoblaciones protectoras, en las que una gestión inadecuada compromete la estabilidad de las masas, las debe convertir en asunto prioritario. Su edad, aunque algo alejada de los turnos habituales, no es impedimiento para la aplicación de cortas de regeneración que, además, pueden potenciar la naturalización y resiliencia de estas repoblaciones. Sin embargo, esta opción apenas se ha aplicado en las miles de hectáreas de pino resinero (Pinus pinaster Ait.) situadas al sur del Sistema Central. Por ello, en este trabajo se ha estudiado un cantón de repoblación de pino resinero situado en Fuencaliente (Ciudad Real), con cierto grado de diversificación en el subpiso (distintas especies de Quercus mediterráneos y madroño), que fue cortado a hecho en dos tiempos en 2012. Previamente a las cortas, se distinguieron tres tipos de masa (pinar puro, y dos grados de mezcla con frondosas), instalándose 78 parcelas de regeneración repartidas equilibradamente entre los tres tipos. Estas parcelas se han inventariado anualmente durante 7 años, clasificando el regenerado de las especies arbóreas en cuatro clases de altura, y midiéndose diferentes parámetros ecológicos asociados. La regeneración del pino puede considerarse exitosa tanto en cantidad como en distribución, asociándose positivamente con veranos frescos y húmedos. No obstante, no se ha promovido una diversificación específica apreciable, pues los Quercus sólo regeneran bien donde eran abundantes ya antes de las cortas.

Palabras clave: adaptación, diversificación, cambio global, naturalización, repoblaciones protectoras.

\section{Summary}

The actual context of global change necessary leads to the application of adaptive silviculture techniques in forests. Nevertheless, the particular situation of several protective reforestations, in which an inadequate management jeopardizes its stability, makes them a priority nowadays. Although its ages are still far from rotation ages, regeneration fellings may enhance naturalization and resilience of these reforestations. However, this solution has been scarcely developed in the maritime pine (Pinus pinaster Ait.) forests located at the south of the Central System mountain range. Thus, this work studied a reforested maritime pine stand with variable presence of broad-leaved species under cover located in Fuencaliente (Ciudad Real). This stand, cut following a seed-tree method in 2012, presented before the cuttings three types of forest structure: pure pine forest and two mix levels. 78 circular plots were installed to monitor pine and Quercus regeneration (classified in four height classes) and several ecological parameters. Pine regeneration could be considered as successful in both density and spatial distribution, and was positively associated with humid and warm summer. Specific diversification, however, was not promoted enough, as Quercus regeneration was abundant only in plots with significant densities of adult trees of these species.

Keywords: adaptation, diversification, global change, naturalization, protective reforestations. 


\section{Introducción}

Aunque la falta de superficie forestal empezó a ser considerada un problema a finales del siglo XIX, sería en la segunda mitad del siglo XX cuando el desarrollo del Plan General de Repoblación Forestal de España permitiría abordar con éxito esta problemática. Un total de 3.5 millones de hectáreas (Montero, 1997), la mayoría de ellas con un objetivo protector contra la erosión y ejecutadas con especies heliófilas del género Pinus, fueron repobladas en el marco de este plan. Debido a la ausencia generalizada de selvicultura de mejora, muchas de estas masas tienen comprometidas su estabilidad y persistencia (Madrigal, 1998), situación agravada por el cambio global. En este contexto, García-Güemes y Calama (2015) desarrollaron algunas líneas maestras de una selvicultura adaptativa, cuyos pilares son la diversificación estructural y específica y el aumento de la resistencia de los individuos ante agentes bióticos y abióticos.

La estrategia de diversificación que más se ha desarrollado en España para la naturalización de las repoblaciones ha sido la ejecución de claras de distintas tipologías, buscando intervenir sobre la diversidad estructural del rodal (Montes et al., 2004; Crecente-Campo et al., 2009) o modificar la composición específica del sotobosque (Rodríguez-Calcerrada et al., 2008; Castillo et al., 2013). Sin embargo, el progresivo acercamiento a las edades teóricas de corta justifica abordar la regeneración de muchas de estas repoblaciones, manteniendo su objetivo protector mientras se potencia la naturalización de las mismas.

En el caso del pino resinero (Pinus pinaster Ait.), en los últimos años diversos estudios han aportado valiosa información sobre su regeneración natural tras cortas, si bien han estado centrados casi en exclusiva en las masas naturales de esta especie en la Meseta Norte (Guerra y Bravo, 2004; González-Alday et al., 2009; Rodríguez-García et al., 2011, o Vergarechea et al., 2019a y b). Otra situación que ha sido muy estudiada ha sido la regeneración post-incendio del pino resinero, tanto en masas naturales como repobladas (Madrigal et al., 2005; Calvo et al., 2008).

Si nos centramos ya en masas de repoblación de esta especie, y excluimos la existencia previa de incendios, la literatura es más bien escasa, y en desarrollo actualmente, con trabajos como el de Muñoz Haro (2017) en Poblet, o el de Fernández-Ramírez (2018) en Guadalajara con entresaca por bosquetes. Sin embargo, existen todavía algunas incógnitas en el comportamiento del regenerado de Pinus pinaster, como pueden ser las miles de hectáreas de repoblación situadas al sur del Sistema Central (y más en concreto, las de Sierra Madrona), o aquellas masas que ya presenten cierta diversificación específica, con presencia de frondosas en el subpiso.

Los objetivos concretos de este trabajo fueron:

1. Comprobar la efectividad de las cortas a hecho en dos tiempos en la regeneración de Pinus pinaster Ait.

2. Estudiar el efecto sobre la diversificación específica de este tipo de cortas de regeneración, en relación con la composición inicial. 
3. Evaluar el efecto de diversos factores ecológicos sobre la regeneración de $P$. pinaster y otras frondosas acompañantes.

\section{Material y Métodos}

\subsection{Sitio de estudio}

El trabajo se ha desarrollado en el cantón D2 del M.U.P. nº 1, "Arroyo del Azor" (Fuencaliente, Ciudad Real), propiedad del ayuntamiento de dicho municipio. Se trata de una repoblación con pino resinero ejecutada mediante siembra entre 1946 y 1949 (Motos y Cabrera, 2009), con presencia natural de frondosas en el subpiso en densidades variables.

El rodal de actuación, conocido como "Riñoncillos", tiene una extensión de 42.8 ha, situadas entre los 780 y los $870 \mathrm{~m}$ de altitud, en orientación predominantemente de solana, y con pendientes inferiores al $25 \%$ en su mayoría. En cuanto a las condiciones de la estación, el clima es típicamente mediterráneo, con precipitaciones anuales de $740 \mathrm{~mm}$, concentradas sobre todo en otoño e invierno; y con una temperatura media anual de $15.3^{\circ} \mathrm{C}$. El suelo es un luvisol háplico (Montero et al., 1999).

\subsection{Cortas realizadas}

Durante la primavera-verano de 2012 (cuando la masa tenía alrededor de 65 años) se ejecutó la primera intervención de una corta a hecho en dos tiempos, utilizando cosechadora en el apeo y autocargador en la saca, mediante sistema de madera corta, y extrayéndose también las copas a cargadero. Las frondosas presentes fueron respetadas en su mayoría, mientras que el escaso pino piñonero (Pinus pinea L.) procedente de la repoblación se cortaría en su totalidad, y la densidad de pino resinero se reduciría hasta 10-50 pies $\cdot \mathrm{ha}^{-1}$ (Fig. 1) (Fig. 2).

\subsection{Dispositivo experimental y metodología de muestreo}

Se identificaron tres tipos de masa previamente a la corta: pinar puro ( $>90 \%$ pies $P$. pinaster, con frondosas casi inexistentes), que en adelante llamaremos "Pura"; masa mixta con pocas frondosas (70-90\% pies $P$. pinaster, $<100$ pies $^{\prime} \cdot \mathrm{ha}^{-1}$ frondosas), en adelante "Mixta 1"; y masa mixta con frondosas abundantes $(<70 \%$ P. pinaster, $>100$ pies $\cdot h^{-1}$ frondosas), en adelante "Mixta 2". Se instalaron un total de 26 parcelas circulares de $500 \mathrm{~m}^{2}$ (12,62 $\mathrm{m}$ de radio), repartidas como sigue: 9 en Pura, 9 en Mixta 1 y 8 en Mixta 2.

En cada parcela se realizaron dos inventarios dasométricos de los pies mayores de $7.5 \mathrm{~cm}$ de diámetro normal, uno previo y otro posterior a la corta, para así poder caracterizarla. Para ello, se midieron diámetros normales de todos los pies distinguiendo la especie, así como alturas totales y diámetros de copa de los cinco pinos resineros más gruesos por parcela y de entre dos y cuatro pies de cada especie de 


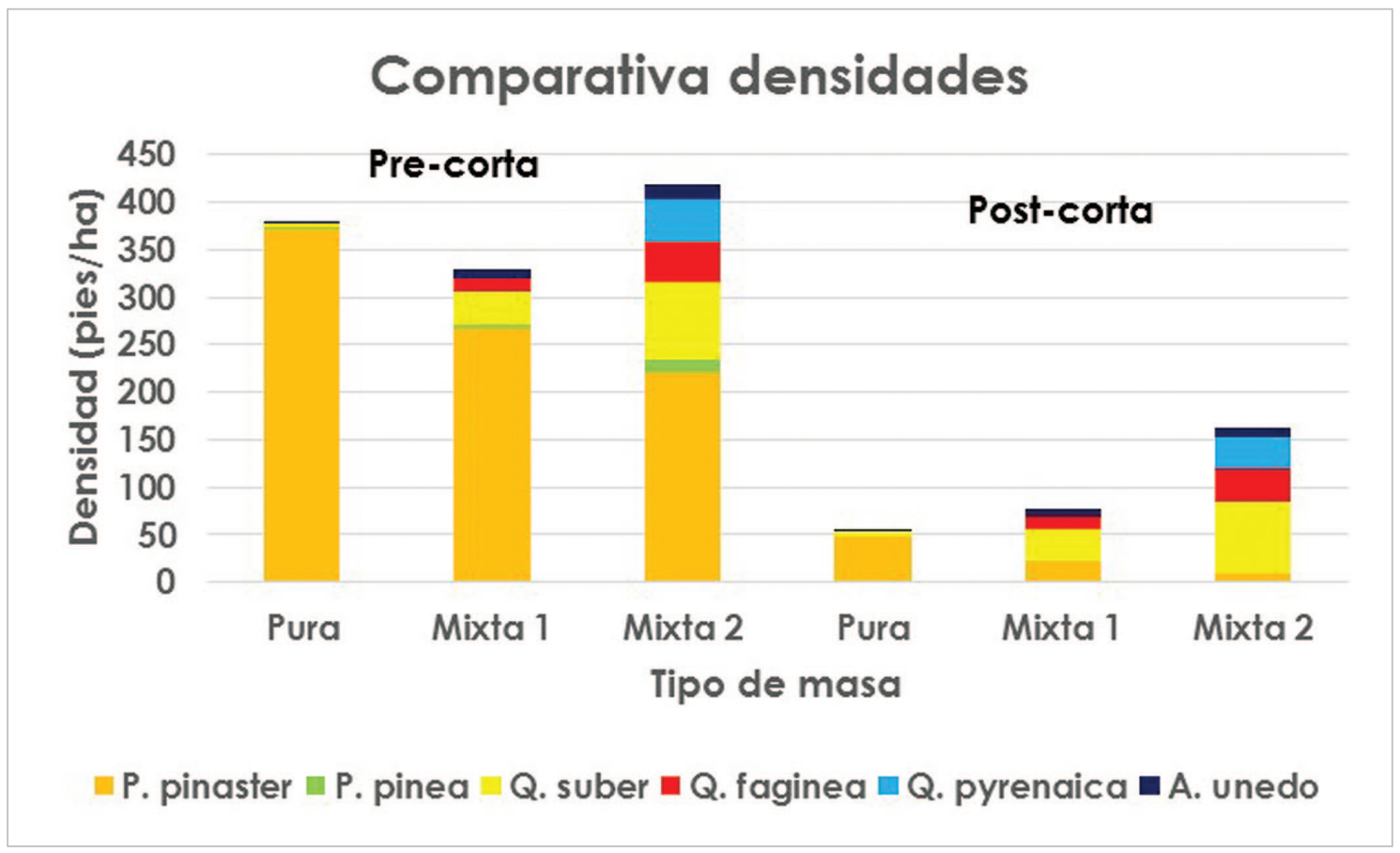

Figura 1. Comparativa de densidades de las especies arbóreas presentes en el cantón entre la situación pre-corta (2011) y la situación post-corta (2012).

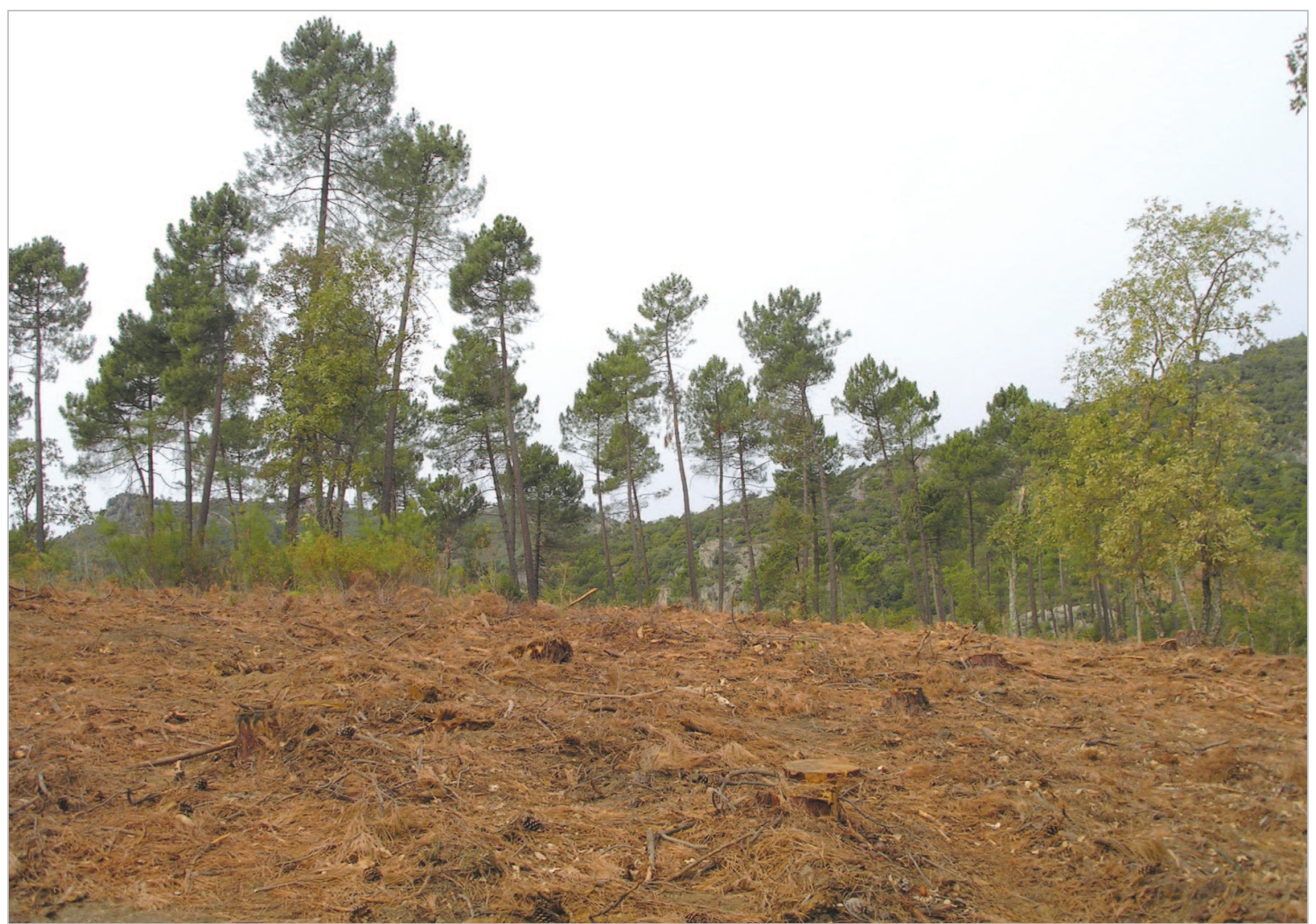

Figura 2. Estado del cantón de actuación después de las cortas de regeneración.

foto: Ricardo Ruiz-Peinado, año 2012. 
frondosa por parcela. A su vez, cada parcela contaba con una subparcela concéntrica de $5 \mathrm{~m}$ de radio, donde se inventariaron todos los pies con $\mathrm{d}<7.5 \mathrm{~cm} \mathrm{y} \mathrm{h}>1.3 \mathrm{~m}$; y tres subparcelas de regeneración de $1 \mathrm{~m}$ de radio, una de ellas concéntrica con la parcela de inventario dasométrico y las otras dos, a $6 \mathrm{~m}$ del centro en las orientaciones Norte y Oeste.

En las subparcelas de regeneración se muestreó, cada otoño entre 2011 y 2018 , el regenerado de . pinaster y de las distintas especies de Quercus spp. (Fig. 3.). El regenerado se clasificó en cuatro clases de altura (0-10, 10-50, 50-90 y 90-130 cm), si bien, a efectos del tratamiento estadístico posterior, el regenerado de pino se reclasificó a plántulas $(0-10 \mathrm{~cm})$ y pimpollos $(>10 \mathrm{~cm})$, al observarse en campo que el regenerado del año no superaba $l o s 10 \mathrm{~cm}$ de altura y que el regenerado mayor de 1 año superaba esa altura siempre. La dificultad para clasificar por especie los ejemplares jóvenes de Quercus y el menor crecimiento de estas plántulas hizo imposible aplicar una división clara por especies y estadíos de desarrollo en este caso. En las subparcelas de regeneración se recogieron también datos sobre la cobertura de matorral, restos vegetales y herbáceas.

\subsection{Análisis estadístico}

El grueso del análisis estadístico se realizó a través de la utilización de coeficientes de correlación de Spearman, valorando así la relación entre el regenerado y las diferentes variables ecológicas analizadas. Esta metodología está muy implantada en estudios de regeneración (Rodríguez-García et al., 2010; Vergarechea et al., 2019a) y permite una visualización sencilla de los resultados. En el caso de la correlación entre la cantidad de regenerado de pino y Quercus, se realizó un filtrado previo de los resultados, eliminando las parejas de datos $(0,0)$ que podrían enmascarar los resultados.

También se utilizaron tablas de contingencia que, a través de la distribución $\chi^{2}$, permiten valorar la existencia de relaciones entre la estructura del arbolado adulto y la composición específica del regenerado (Vergarechea et al., 2019a).

Para todos los análisis se ha empleado el software estadístico R-Studio (R Studio Team, 2019), considerándose como nivel de significación $\alpha=0.05$.

\section{Resultados y Discusión}

\subsection{Dinámica temporal del regenerado e influencias del clima}

Podemos observar la dinámica temporal del regenerado de $P$. pinaster en las $f i$ guras $4 a, 4 b, 4 c$, distinguiendo por clases de altura y año. Para los tres tipos de masa preexistente, podemos observar cómo el efecto de la corta es muy importante en la reducción de densidades, que eran elevadas previamente a la corta y con mayoría de pimpollos (aunque desconocemos cuál era la viabilidad de este regenerado), y caen drásticamente con la corta. En 2013, un año después de las cortas, se observa un fuerte incremento en el número de plántulas (aprovechando un año cli- 


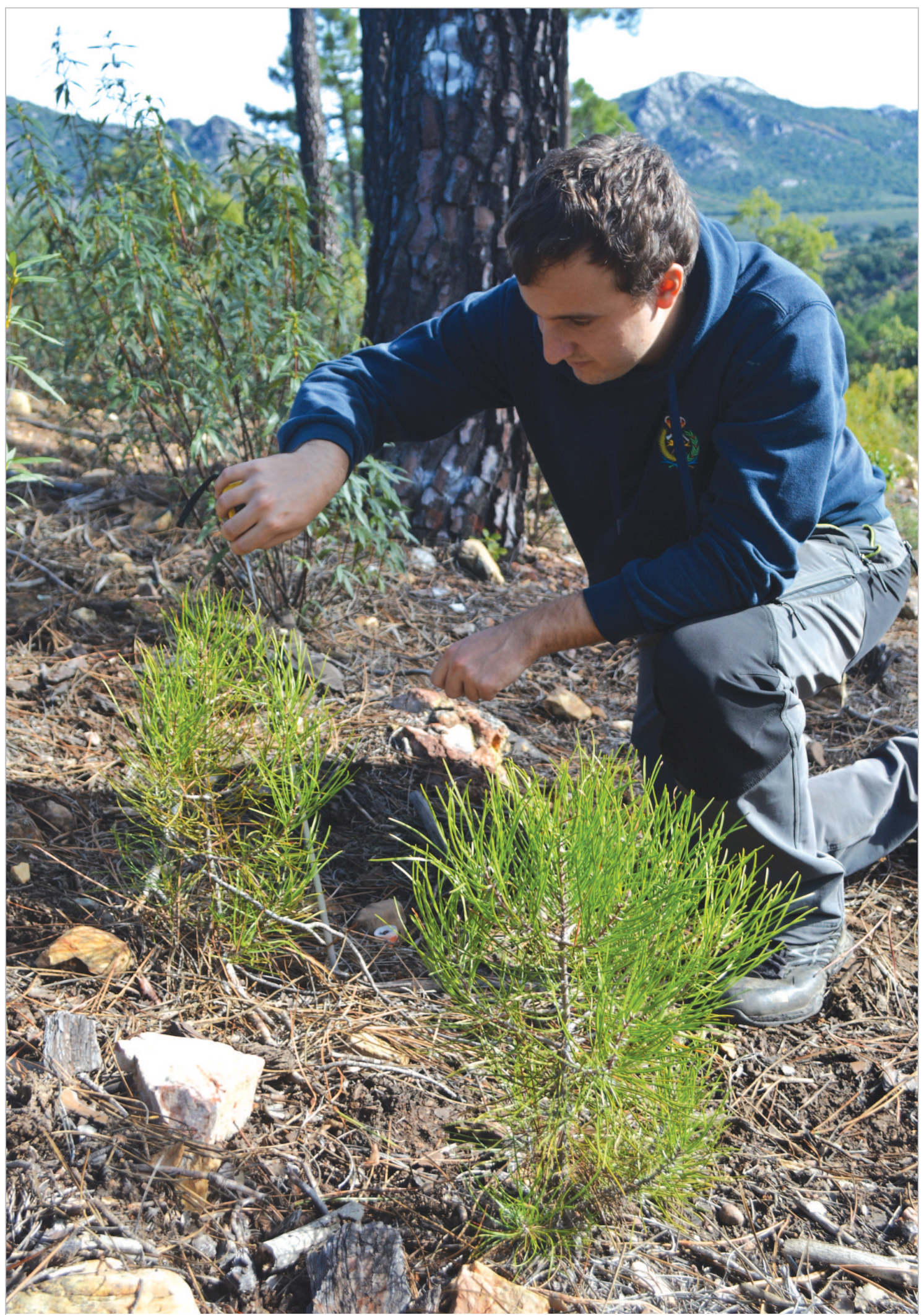

Figura 3. Medición de la altura de pimpollos, realizada con flexómetro. 
matológicamente favorable), que se van estableciendo en los años posteriores, alcanzando en 2018 niveles similares a los previos a la corta, tanto en densidad como en proporción de pimpollos. Si comparamos los tres tipos de masa entre sí, podemos ver como las densidades son siempre mayores en la masa Pura, si bien en los tres casos se puede considerar exitosa al superar los 2000 pies ha ${ }^{-1}$ que ponen como referencia Rodríguez-Soalleiro et al. (2008).
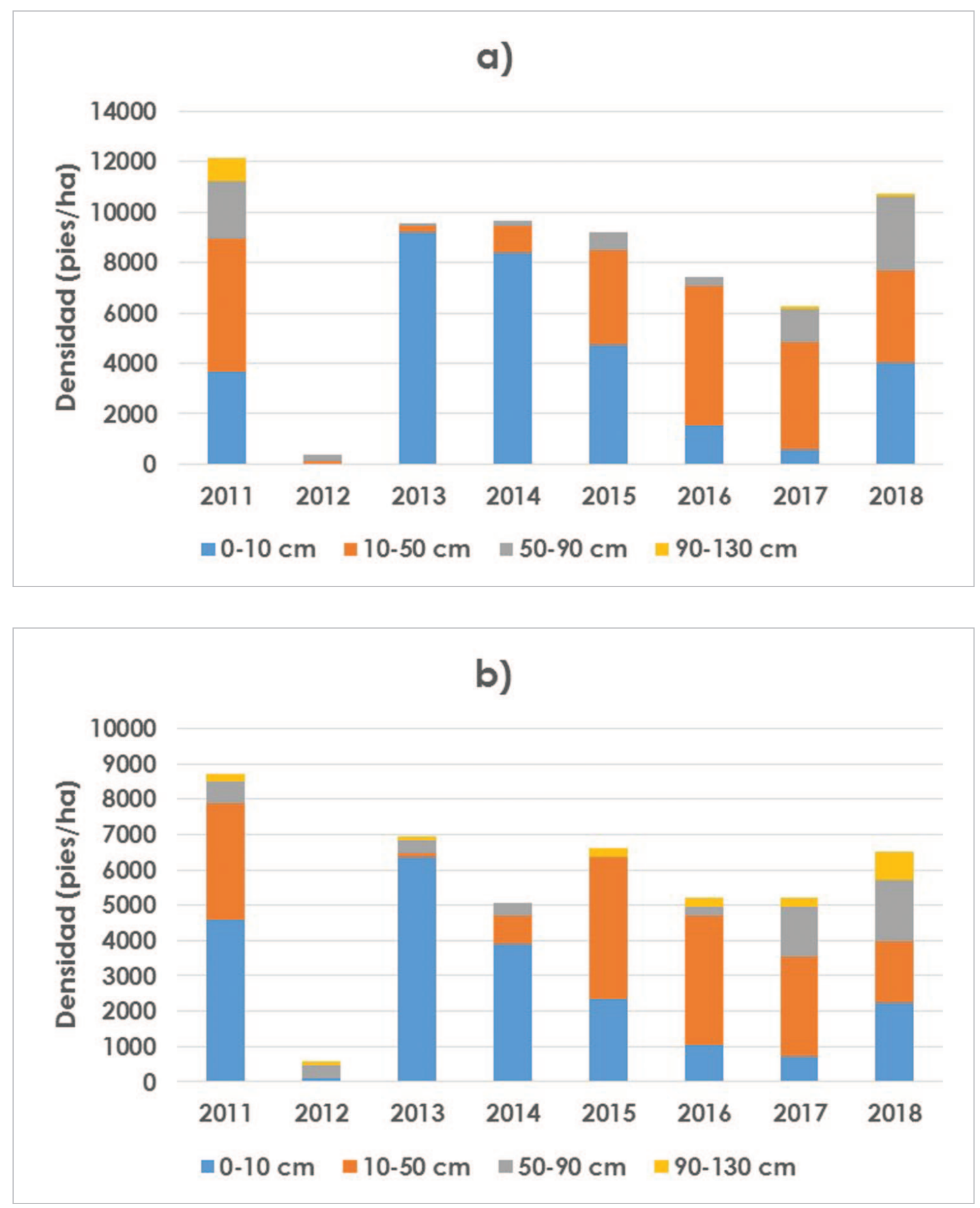


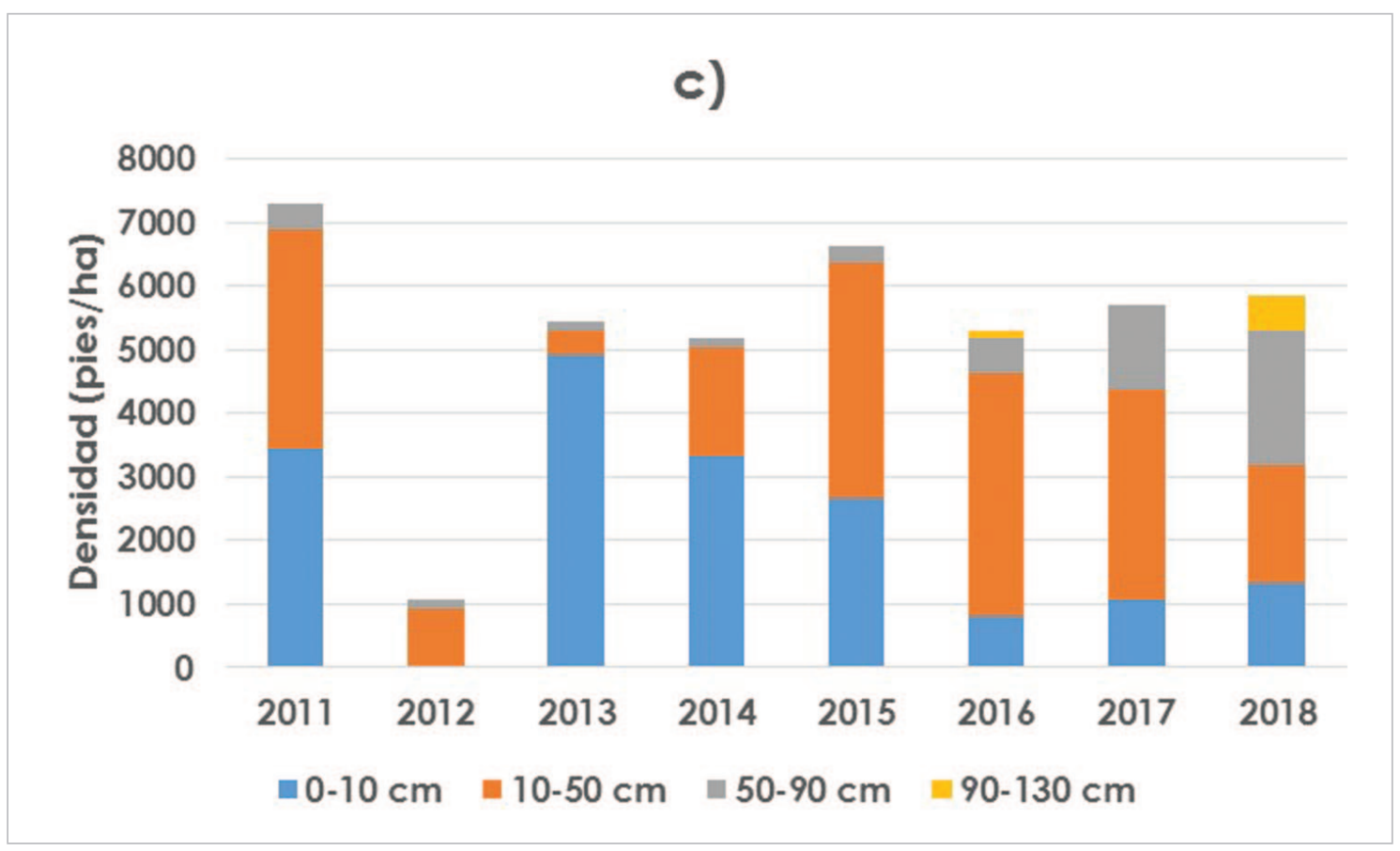

Figura 4. Densidad de regenerado de pino resinero (P. pinaster) clasificado por clases de altura y año de inventario en a) masa Pura, b) masa Mixta 1 y c) masa Mixta 2.

En el caso de Quercus spp., como se puede observar en la figura 5, la dinámica es algo diferente. Igualmente encontramos máximos de regeneración previos a la corta, que caen drásticamente por efecto de ésta. Sin embargo, tras la ejecución de la corta, se producen diferentes situaciones según el tipo de masa. En la masa pura, se produce una incorporación importante en 2013, que muere en su mayoría antes de la siguiente medición, manteniéndose desde entonces más o menos estable la densidad. En cambio, en la masa Mixta 1, aunque la incorporación de 2013 se mantiene en 2014, al año siguiente la densidad cae ya a niveles similares a los actuales. Por su parte, en la masa Mixta 2, se está produciendo una incorporación más gradual tras la corta, presentando actualmente los valores máximos de los tres tipos de masa, y acercándose a los niveles previos a la corta.

Al relacionar estas dinámicas con los datos climáticos (Tabla 1), se observa que las precipitaciones de septiembre y verano son positivamente significativas para la emergencia de plántulas, mientras que para la cantidad de pimpollos (que incluye tanto el establecimiento de plántulas como la propia mortalidad de pimpollos), influyen negativamente las temperaturas máximas de agosto y septiembre. La influencia positiva de la precipitación estival en la emergencia de plántulas de $P$. pinaster ha sido destacada por Ruano et al. (2009) y Rodríguez-García et al. (2011). También Calama et al. (2017) señalan que la lluvia estival y una liberación progresiva de la cubierta son vitales para la emergencia y supervivencia del regenerado de pino resinero. Además, Vergarechea et al. (2019b) encontraron que, para el regenerado ya establecido en pinares mediterráneos, los veranos con temperaturas máximas muy elevadas provocan una mayor mortalidad. No se encontraron factores climáticos 


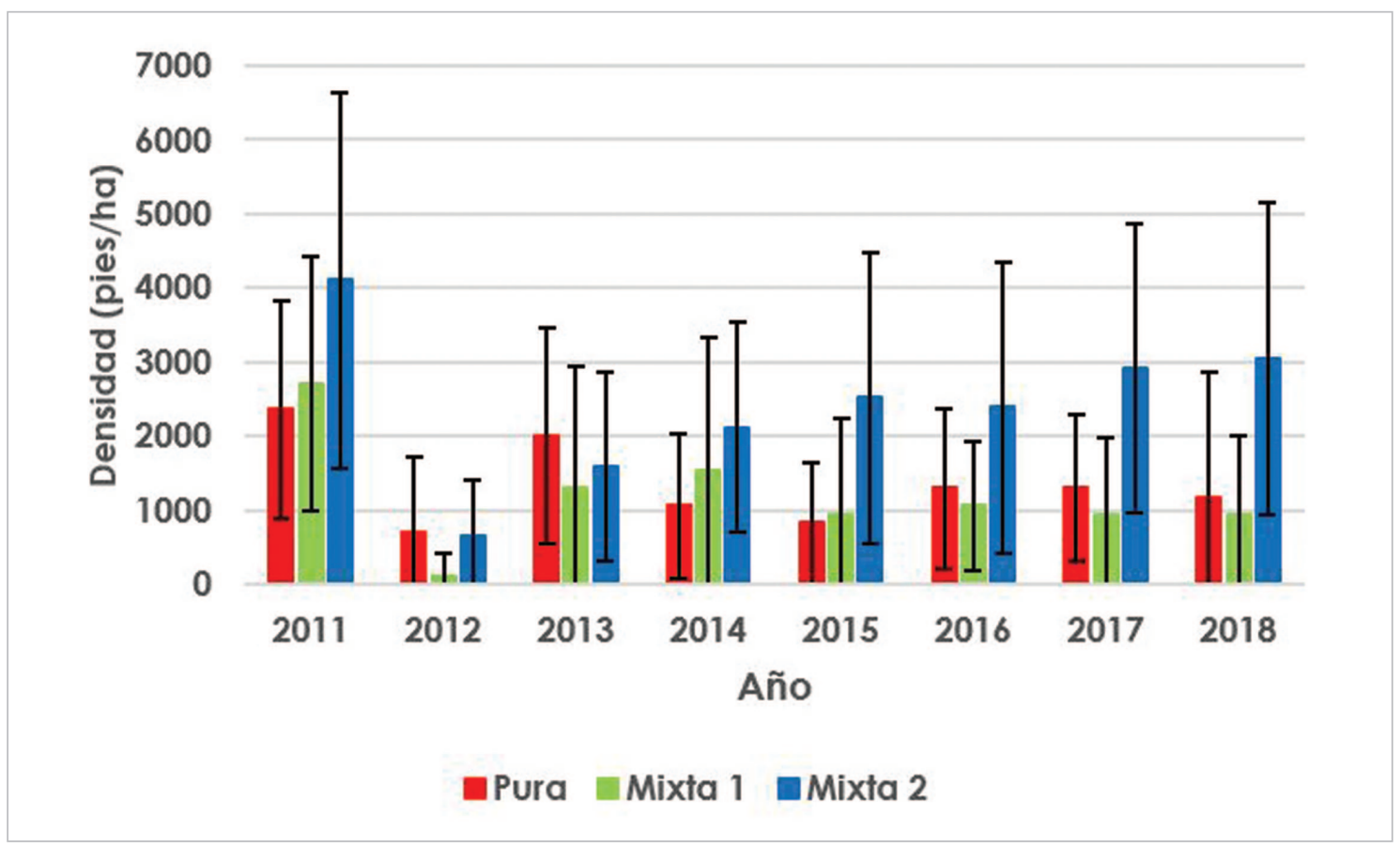

Figura 5. Densidad de regenerado de Quercus spp. por tipo de masa y año,

Tabla 1. Correlaciones climáticas significativas ( $\mathrm{p}$-value $<0.05$ ) encontradas en relación al regenerado de P. pinaster. Abreviaturas: $\mathrm{P}$ (precipitación), T (temperatura), max (máximas), AGO (agosto) y SEPT (septiembre).

\begin{tabular}{ccc}
\hline & P.pinaster plántulas & P.pinaster pimpollos \\
\hline PtotalVERANO & 0.81 & \\
\hline Psept & 0.86 & \\
\hline TmaxAGO & & -0.9 \\
\hline TmaxSEPT & & -0.82 \\
\hline
\end{tabular}

significativos para la regeneración de Quercus spp., lo que podría indicar que son otros factores, como la disponibilidad o predación de la bellota y plántula, los que están influenciando su dinámica temporal.

\subsection{Distribución espacial del regenerado}

La aplicación de tablas de contingencia sobre la presencia del regenerado de $P$. pinaster y Quercus spp. arroja que la presencia de regenerado de $P$. pinaster, y más concretamente, de "seedlings", depende de la estructura y composición específica del estrato de árboles adultos (Tabla 2), indicando que esta especie regenera peor en masas con menor proporción de pies adultos conespecíficos, un resultado concordante con el encontrado por Vergarechea et al. (2019a). 
De la misma manera, se encontró una correlación significativa negativa (-0.39, p-value $<0.05)$ entre las densidades de P. pinaster y Quercus spp., indicando que ambas "especies" tienen distintos requerimientos de instalación, sobre todo relacionados con la iluminación del suelo.

Tabla 1. Tabla de contingencia para la presencia/ausencia de regenerado según tipo de masa en el año 2018 (último año de inventario). Valores en negrita indican valores observados, en cursiva valores esperados, $\mathrm{y} *$ indica significación estadística ( $\mathrm{p}$-value $<0.05$ ).

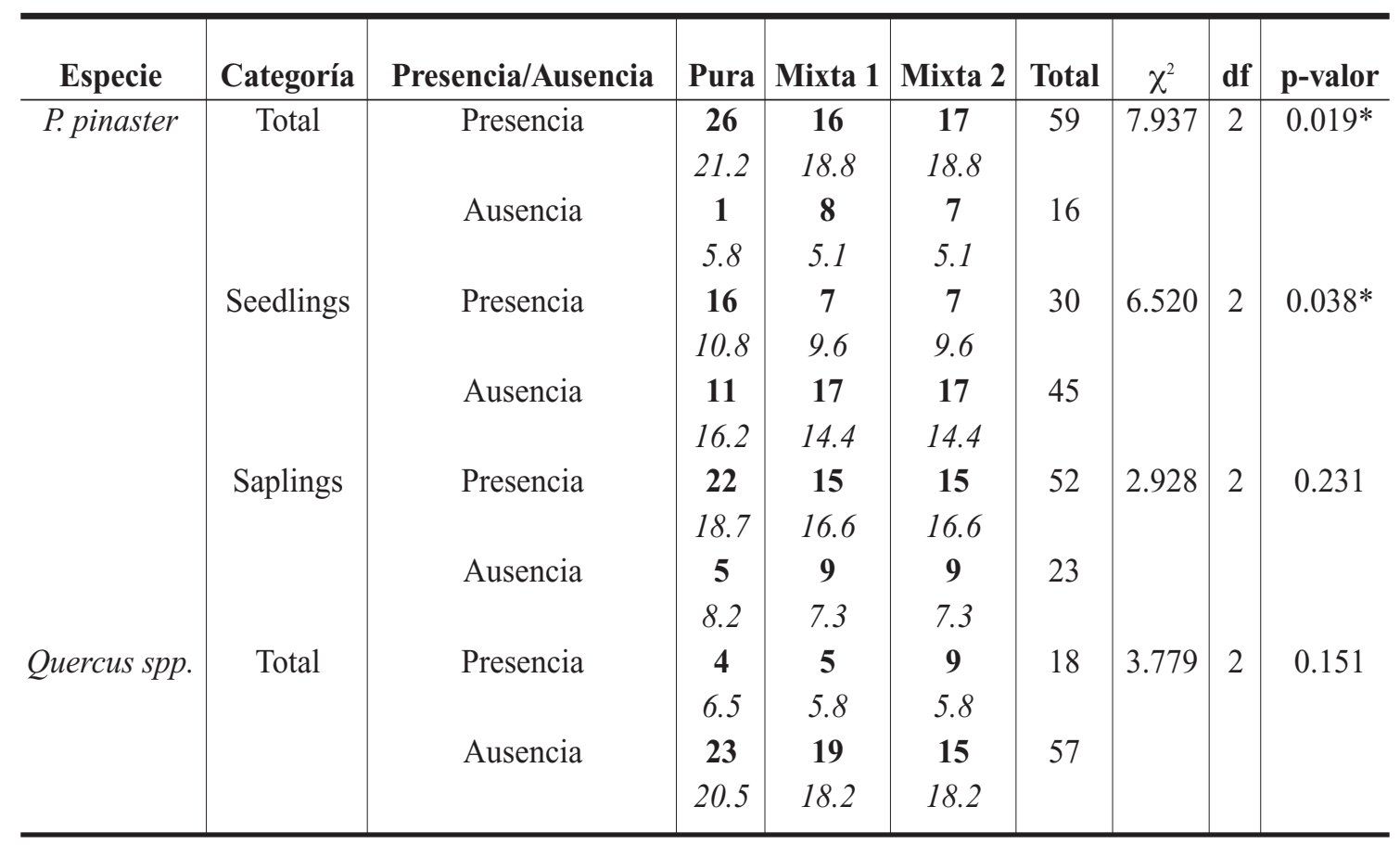

En cuanto a las relaciones con parámetros fisiográficos los coeficientes de correlación de Spearman señalaron correlaciones significativamente negativas entre las densidades de regenerado de pino y la pendiente $(\mathbf{- 0 . 4 5}$, p-value $<0.05)$, y entre las densidades de Quercus y la cota (-0.4, p-value <0.05). En el caso del pino, el trabajo de Muñoz Haro (2017) ya había señalado que una mayor pendiente implicaba un mayor arrastramiento de la semilla y una menor profundidad de suelo, lo que influía negativamente en la densidad de regenerado. Creemos que la relación entre regenerado de Quercus y cota se explica indirectamente por humedad disponible: en el dispositivo estudiado, menores cotas suponen posiciones inferiores en la ladera, con mayor acumulación de humedad que puede favorecer el desarrollo de la frondosa. No se encontraron relaciones significativas con otros parámetros ecológicos como la cobertura de herbáceas, restos vegetales o matorral, pese al enorme crecimiento y distribución de éste, como se observa en la figura 6, que nos muestra la situación actual del cantón regenerado. 


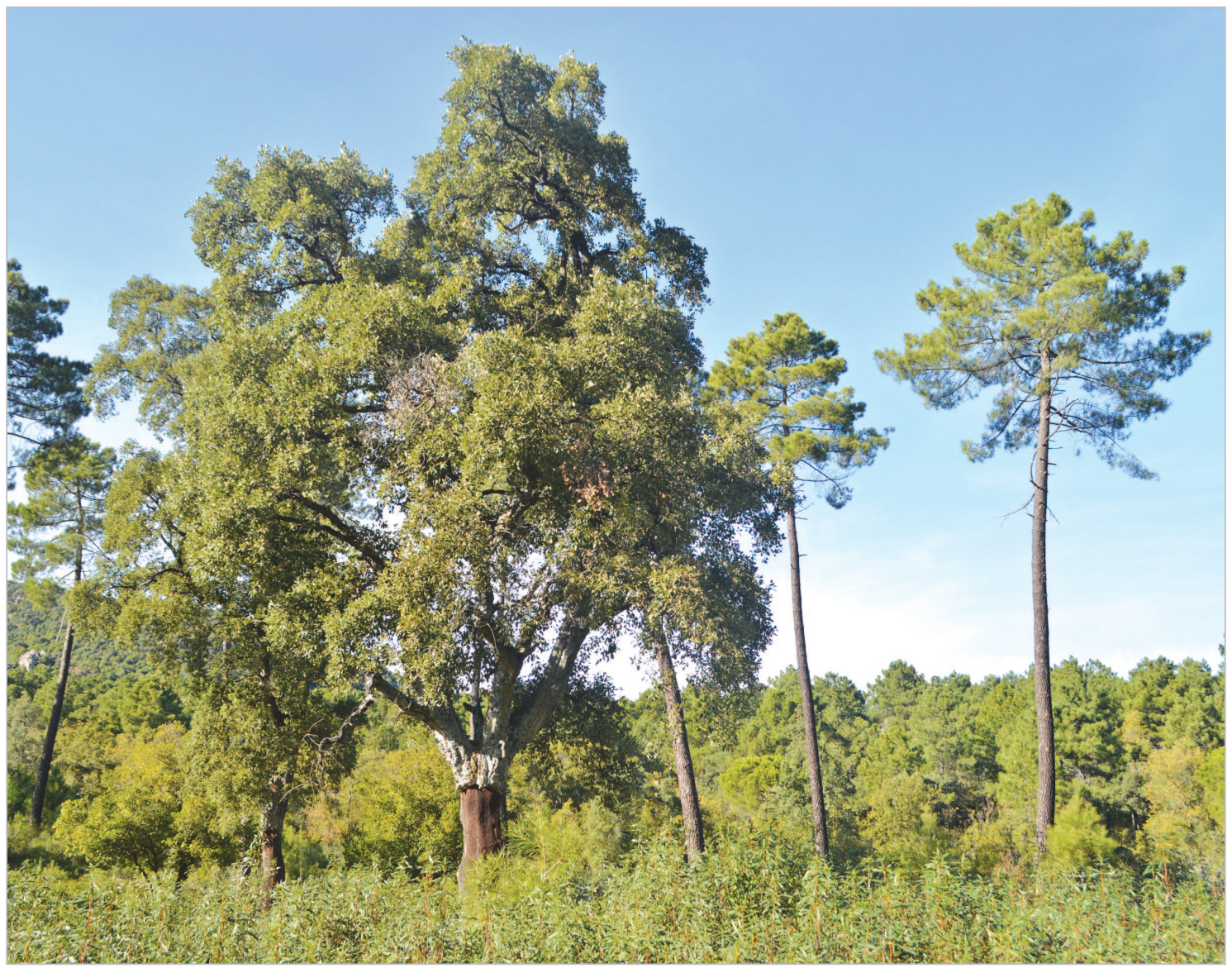

Figura 6. Estado del cantón en el año 2018, con abundante matorral heliófilo (sobre todo diferentes especies de cistáceas). En primer término, un alcornoque (Quercus suber) descorchado, junto a pies remanentes de pino resinero.

\section{Conclusiones}

Aunque actualmente podamos considerar que la masa principal de pino resinero ha regenerado exitosamente, la presencia de menores densidades de regenerado tanto de pino como de frondosas con respecto a la situación previa a la corta (aun desconociendo su viabilidad) parece indicar que una actuación menos intensa podía haber tenido mayor éxito en la regeneración del cantón estudiado, evitando la invasión de matorral heliófilo y favoreciendo la diversificación específica de la masa al disminuir daños sobre los Quercus preexistentes.

Igualmente, hemos podido comprobar como en una masa situada más al sur que las habitualmente estudiadas, veranos climatológicamente más favorables (más húmedos y menos calurosos) permiten la supervivencia de una mayor cantidad de pimpollos. 


\section{Agradecimientos}

Este trabajo se ha llevado a cabo gracias a la colaboración entre la Escuela de Ingeniería de Montes, Forestal y del Medio Natural de la Universidad Politécnica de Madrid y el INIA-CIFOR. La financiación para la instalación del ensayo y las posteriores mediciones ha sido proporcionada por los proyectos AGL2011-29701-C0201, AGL2014-51964-C2-2-R y AGL2017-83828-C2.1R. Se quiere agradecer también la colaboración y apoyo del Servicio de Montes de Ciudad Real, la cesión de los datos climáticos a AEMET, y la imprescindible colaboración de diferentes técnicos del INIA-CIFOR en el trabajo de campo.

\section{Referencias bibliográficas}

Calama, R.; Manso, R.; Lucas-Borja, M. E.; Espelta, J. M.; Piqué, M.; Bravo, F.; del Peso, C.; Pardos, M.; 2017. Natural regeneration in Iberian pines: A review of dynamic processes and proposals for management. For. Syst., 26 (2), eR02S. https://doi.org/10.5424/fs/201726211255

Calvo, L.; Santalla, S.; Valbuena, L.; Marcos, E.; Tárrega, R.; Luis-Calabuig, E.; 2008. Post-fire natural regeneration of a Pinus pinaster forest in NW Spain. Plant. Ecol., 197(1), 81-90. https://doi.org/10.1007/s11258-007-9362-1

Castillo, V.M.; Barberá, G.G.; Querejeta, J.I.; Martínez-Sánchez, M.A.; Martínez-Fernández, F.; 2009. Diversificación de masas repobladas de pino carrasco mediante claras e introducción de sotobosque. Act. $5^{\circ}$ Congr. For. Esp. Ávila.

Crecente-Campo, F.; Pommerening, A.; Rodríguez-Soalleiro, R.; 2009. Impacts of thinning on structure, growth and risk of crown fire in a Pinus sylvestris L. plantation in northern Spain. For. Ecol. Manage., 257(9), 1945-1954. https://doi.org/10.1016/j.foreco.2009.02.009

Fernández Ramírez, S.; 2018. Regeneración tras cortas a hecho por bosquetes sobre repoblaciones de Pinus pinaster Ait. ssp. mesogeensis en la Sierra Norte (Guadalajara). Trabajo Fin de Grado en Ingeniería Forestal. ETSI Montes, Forestal y del Medio Natural. Universidad Politécnica de Madrid. http://oa.upm.es/52962/

García-Güemes, C.; Calama, R.; 2015. La práctica de la selvicultura para la adaptación al cambio climático. En: Herrero, A.; Zavala, M.A.; (Eds.): Los bosques y la biodiversidad frente al Cambio Climático: Impactos, Vulnerabilidad y Adaptación en España, pp. 501-512

González-Alday, J; Martínez-Ruiz, C; Bravo F; 2009. Evaluating different harvest intensities over understory plant diversity and pine seedlings, in a Pinus pinaster Ait. natural stand of Spain. Plant. Ecol., 211-220. https://doi.org/10.1007/978-90-481-2795-5_16

Guerra, B.; Bravo, F.; 2004. Análisis y modelización de la regeneración de Pinus pinaster

Ait. en el Sistema Ibérico Meridional. Cuad. Soc. Esp. Cienc. For., 18, 167-172

Madrigal, A.; 1998. Problemática de la ordenación de masas artificiales en España. Cuad. Soc. Esp. Cienc. For., 6, 13-20.

Madrigal, J.; Hernando, C.; Martínez, E.; Díez, C; y Guijarro, M.; 2005. Regeneración post-incendio de Pinus pinaster Ait. en la Sierra de Guadarrama (Sistema Central, España): modelos descriptivos de los factores influyentes en la densidad inicial y la supervivencia. Invest. Agr. Sist. Rec. For., 14, 36-51 
Montero, G.; 1997. Breve descripción del proceso repoblador en España (1940-1995). Legno Celulosa Carta, 4, 35-42.

Montero, G.; Ortega, C.; Cañellas, I.; Bachiller, A.; 1999. Productividad aérea y dinámica de nutrientes en una repoblación de Pinus pinaster Ait. sometida a distintos regímenes de claras. Invest. Agr. Sist. Rec. For., fuera de serie no 1, 175-206

Montes, F.; Cañellas, I.; del Río, M.; Calama, R.; Montero, G.; 2004. The effects of thinning on the structural diversity of coppice forests. Ann. For. Sci., 61, 771-779. https://doi.org/10.1 051/forest:2004074

Motos, J.; Cabrera, M.; 2009. Proyecto de Ordenación Integral del Grupo de montes de Fuencaliente (Ciudad Real)

Muñoz Haro, E.; 2017. Dinámica inicial del regenerado en masas de pino rodeno (Pinus pinaster Ait.) del Paraje Natural de Interés Nacional de Poblet: recomendaciones silvícolas frente a nuevos escenarios de gestión. Trabajo Fin de Grado en Ingeniería Forestal. Universidad de Lleida.

Rodríguez-Calcerrada, J.; Mutke, S.; Alonso, J.; Gil, L.; Pardos, J.A.; Aranda, I.; 2008. Influence of overstory density on understory light, soil moisture, and survival of two underplanted oak species in a Mediterranean montane Scots pine forest. Invest. Agrar. Sist. Recur. For., 17, 31-38. https://doi.org/10.5424/srf/2008171-01021

Rodríguez-García, E.; Juez, L.; Bravo, F.; 2010. Environmental influences on post-harvest natural regeneration of Pinus pinaster Ait. in Mediterranean forest stands submitted to the seed-tree selection method. Eur. J. For. Res., 129(6), 1119-1128. https://doi.org/10.1007 /s10342-010-0399-7

Rodríguez-García, E.; Gratzer, G.; Bravo, F.; 2011. Climatic variability and other site factor influences on natural regeneration of Pinus pinaster Ait. in Mediterranean forests. Ann. For. Sci., 68, 811-823. https://doi.org/10.1007/s13595-011-0078-y

Rodriguez-Soalleiro, R.; Serrada, R.; Lucas, J.A.; Alejano, R.; del Río, M.; Torres, E.; Cantero, A.; 2008. Selvicultura de Pinus pinaster Ait. subsp. mesogeensis. En: Montero, G.; Serrada, R.; Reque, J.A.; (Eds.), Compendio de selvicultura aplicada en España., INIA, Madrid, pp. 399-430.

Ruano, I.; Pando, V.; Bravo, F.; 2009. How do light and water influence Pinus pinaster Ait. germination and early seedling development? For. Ecol. Manage., 258, 2647-2653. https://doi. org/10.1016/j.foreco.2009.09.027

Vergarechea, M.; del Río, M.; Gordo, J.; Martín, R.; Cubero, D; Calama, R; 2019a. Spatio-temporal variation of natural regeneration in Pinus pinea and Pinus pinaster Mediterranean forests in Spain. Eur. J. For. Res., 138, 313-326. https://doi.org/10.1007/s10342-019-01172-8

Vergarechea, M.; Calama, R.; Fortin, M.; del Río, M; 2019b. Climate-mediated regeneration occurrence in Mediterranean pine forests: A modeling approach. For. Ecol. Manage., 446, 10-19. https://doi.org/10.1016/j.foreco.2019.05.023 